\title{
2021 \\ Erste israelisch-deutsche Mathematikerkonferenz in Jerusalem
}

\author{
Kristina Vaillant
}

\begin{abstract}
Binationale Konferenzen hat die Deutsche Mathematiker-Vereinigung schon mit zahlreichen Ländern gemeinsam organisiert. Aber die israelisch-deutsche Konferenz, zu der die Israel Mathematical Union gemeinsam mit der DMV vom 8. bis 10. März 2021 nach Jerusalem einlädt, ist eine Premiere. Gastgeber ist die Hebrew University. Dort lehrt und forscht Alex Lubotzky, Präsident der Israel Mathematical Union. Mit ihm habe ich noch vor Ausbruch der Corona-Pandemie via Skype über die Beziehungen zwischen beiden Ländern in der Mathematik gesprochen und darüber, was er sich von der Konferenz erhofft.
\end{abstract}

Herr Lubotzky, als ich Ihnen den 27. Januar 2020 als Termin für unser Interview vorschlug, war mir nicht bewusst, dass das der Tag des Gedenkens an die Befreiung des Konzentrationslagers Auschwitz vor 75 Jahren ist.

Ich habe das auch heute Morgen plötzlich bemerkt, das hat wirklich symbolische Bedeutung.

Ich möchte mein tiefes Mitgefühl für die Opfer des Holocaust ausdrücken. Seien Sie versichert, dass ich als Bürgerin dieses Landes, als Journalistin und als Mutter immer dafür einstehen werde, dass diese Verbrechen nicht vergessen werden und dass ich mich immer gegen jegliche Form des Antisemitismus und Rassismus wenden werde.

Vielen Dank für Ihre freundlichen Worte. Es ist wichtig darüber zu sprechen, denn wann immer es um ein gemeinsames Treffen von Israelis und Deutschen geht, haben wir diese Fragen im Hinterkopf. Und weil Sie so nett sind, möchte ich Ihnen und Ihren Lesern eine persönliche Geschichte erzählen. Meine Eltern sind beide Überlebende des Holocaust. Bei uns zu Hause wurden niemals Waren aus Deutschland eingekauft, mein Vater hat sogar Entschädigungszahlungen abgelehnt, und er ist auch niemals nach Deutschland gereist. Als ich 1979 meine Promotion abgeschlossen hatte, lud mich Otto Kegel nach Oberwolfach an das Mathematische Forschungsinstitut ein. Er widmete das Gruppentheorie-Treffen in dem Jahr den profiniten Gruppen, und das war mein Thema. Ich wollte die Einladung unbedingt annehmen, aber ich zögerte, in erster Linie wegen meines Vaters. Ich rechnete damit, dass er sich entweder sehr aufregen oder dass er sagen würde, okay, das ist dein Leben, mach' was Du willst. Seine Reaktion hat mich dann verblüfft. Er freute sich nämlich sehr. Ich heiße Alexander Lubotzky - nach meinem Großvater, der im Holocaust getötet wurde - und für meinen Vater, der als Partisan in Vilnius gekämpft und den Holocaust überlebt hat, war das wie ein Sieg, dass die Deutschen jetzt anerkennen, dass sie Alexander Lubotzky brauchen. In gewisser Weise war das eine schwierige Ein- führung in unser eigentliches Thema, aber ich halte das für wichtig.

Also zur Konferenz - wir verfolgen damit in allererster Linie wissenschaftliche Ziele, außerdem wollen wir die Beziehungen stärken. Wie Sie wissen, sind beide Länder echte Imperien, wenn es um Mathematik geht. Trotzdem sind die Beziehungen zu den USA momentan viel enger. Auch wenn sich das gerade ein bisschen ändert, in meiner Generation gingen fast alle als Postdoc in die USA. Vielleicht ein paar nach England, einige wenige nach Frankreich, aber fast niemand nach Deutschland. Das liegt teils an der Geschichte und teils an der Sprachbarriere, aber ich denke, die Beziehungen könnten enger sein. In der Geschichte der Beziehungen zwischen Juden und Deutschen gab es Liebe und Hass, und wir ziehen es vor, an die Zeiten der Liebe anzuknüpfen.

Wie sind Sie auf die Idee gekommen, eine israelischdeutsche Konferenz auszurichten?

Ehrlich gesagt ist die Idee nicht neu, darüber wurde in den letzten Jahren immer mal wieder gesprochen. Im Sommer 2018 beim Internationalen Mathematiker-Kongress (ICM) in Rio des Janeiro habe ich Michael Röckner getroffen, der damals Präsident der DMV war, wir haben uns angefreundet, uns unterhalten und dann sind wir eben auch auf diese Idee zu sprechen gekommen. Ich möchte ihm dafür danken, dass er diese Konferenz initiiert hat, und ich möchte auch dem derzeitigen Präsidenten Friedrich Götze danken, genauso wie seinem Stellvertreter Klaus Hulek, der sehr stark in die Organisation involviert ist.

Was können Sie uns zu den inhaltlichen Schwerpunkten der Konferenz sagen?

Es gibt eigentlich keinen Schwerpunkt, das Programm ist breit und deckt mehr oder weniger alle Gebiete der Mathematik ab. Es wird - in der Regel morgens - mehrere Plenarvorträge zu den großen Gebieten der Mathematik geben. Wichtiger sind aber die Sessions am Nachmittag, 
wenn die Teilnehmer unter bis $\mathrm{zu} 20$ verschiedenen Fachvorträgen wählen können: mit Themen von reiner Mathematik wie Zahlentheorie oder algebraische Geometrie bis zu angewandter Mathematik, zum Beispiel in der Materialforschung, aber auch Mathedidaktik und theoretische Informatik. Vorschläge von Mathematikern aus Deutschland für weitere „Special Sessions" nehmen wir noch an, und wir unterstützen sie gerne bei der Umsetzung. Wir erwarten, dass sich gerade aus diesen Gruppen heraus dann auch langfristige Kooperationen ergeben.

Wie kann man sich für diese „Special Sessions“ am Nachmittag bewerben, gibt es einen Anmeldeschluss?

Das handhaben wir äußerst flexibel und unbürokratisch. Man kann direkt an Zeev Rudnick (Tel Aviv University) oder an Thomas Peternell (Universität Bayreuth) schreiben, beide sind Vorsitzende des Programmkommitees, aber genauso gut kann man sich auch an mich wenden.

Gibt es Gebiete, auf denen die Zusammenarbeit zwischen israelischen und deutschen Mathematikern schon heute besonders eng ist?

Das Gebiet der Zahlentheorie ist ein Beispiel dafür. Moshe Jarden von der Tel Aviv University, der das Organisationskommitee leitet, hat viele ehemalige Studierende, die an Universitäten in Israel lehren und die enge Beziehungen zu Mathematikern in Deutschland pflegen. Es wird auch Fachvorträge zu diesem Gebiet geben, selbst wenn das nicht unbedingt notwendig ist, weil die ihre eigenen Fachkonferenzen haben. Ich möchte mit dieser Konferenz vor allem diejenigen Mathematiker motivieren, die noch keine oder nur lockere Verbindungen nach Israel haben. Ob die Konferenz ein Erfolg war, wird sich für mich nicht nach den drei Konferenztagen zeigen, sondern erst nach zwei oder drei Jahren. Der wahre Erfolg sind die Kooperationen, die sich aus der Konferenz ergeben werden.

Wie ist die Resonanz unter israelischen Mathematikern auf diese erste binationale Konferenz?

Die Leute hier freuen sich sehr darauf, Gastgeber zu sein und die Mathematiker aus Deutschland zu empfangen. Wir glauben, dass die Konferenz uns großartige Gelegenheiten bietet, und ich denke, das gilt genauso für die deutsche Seite. Israel - und da bin ich jetzt ganz unbescheiden, denn es geht ja nicht um mich persönlich, sondern um meine Heimat ist nicht nur als Hightech- und Startup-Nation berühmt, sondern auch für seine Leistungen in der Mathematik. Wir haben hier eine starke mathematische Tradition und eine hohe Achtung vor der Mathematik.

Bei der Vorbereitung des Interviews bin ich auf die Geschichte des Instituts für Mathematik an der Hebrew University gestoßen, die bis in die 1920 er Jahre zurückreicht. Edmund Landau von der Universität Göttingen - er war ausgerechnet 1921 auch Präsident der DMV war einer der Gründungsväter ...

Er war der Gründungsvater.

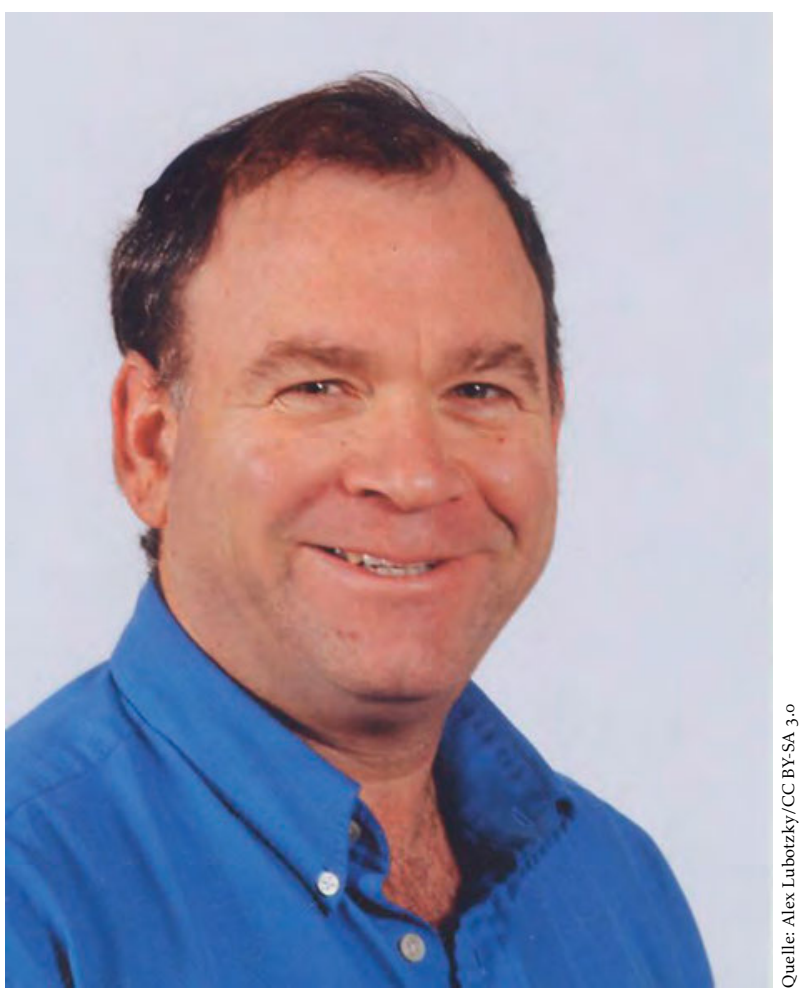

Alex Lubotzky

Wird diese Geschichte Ihres Instituts auch thematisiert?

Daran haben wir bisher nicht gedacht, aber das scheint mir eine gute Idee zu sein. Ehrlich gesagt war ich ein wenig überrascht, als die deutsche Seite darum bat, einen Abendvortrag über jüdische Mathematiker in Deutschland vor und während des Holocaust zu veranstalten. Wir wollten nicht vorpreschen bei dem Thema, aber es war ihr ausdrücklicher Wunsch und selbstverständlich kommen wir dem nach. Leo Corry von der Tel Aviv University ist Experte auf diesem Gebiet, er wird einen Plenarvortrag dazu halten. Und dieser Abend wird dann auch Gelegenheit bieten, über die Geschichte der Mathematik in Israel zu sprechen. Denn sie ist stark durch die Verbindung mit Deutschland geprägt. Und es ist so, wie ich es zu Beginn unseres Gesprächs gesagt habe: Wir wollen dahin zurück, wir wollen so enge Beziehungen wie in den guten alten Zeiten.

\section{Wo wird die Konferenz stattfinden?}

Wir treffen uns auf dem Campus Givat Ram der Hebrew University. Der ursprüngliche Campus lag auf dem Berg Skopus auf der anderen Seite von Jerusalem. Aber der Waffenstillstand von 1948, der das Ende des Unabhängigkeitskriegs Israels markierte, regelte, dass die Truppen dort bleiben, wo sie sich zu diesem Zeitpunkt befanden. Damals war der Campus unter israelischer Kontrolle, aber auf allen Seiten umgeben von jordanischen Truppen. Daher einigte man sich darauf, dass Angehörige der Uni alle zwei Wochen zwischen Jerusalem und dem Campus pendeln. Das galt zwei 
Jahrzehnte lang, aber natürlich kann ein Universitätsbetrieb auf Dauer so nicht aufrechterhalten werden. Also wurde ein neuer Campus eröffnet. Und nach dem Sechstagekrieg, als Israel erneut die Kontrolle über das Gebiet übernommen hatte, bezogen die Geistes- und Sozialwissenschaften den Campus auf dem Skopus-Berg.

Der „neue“ Campus Givat Ram ist den Naturwissenschaften vorbehalten und liegt mitten in der Stadt.

Ja, das Stadtzentrum ist ganz in der Nähe. Und, wissen Sie, Jerusalem ist eine der interessantesten Städte der Welt. Daher hoffen wir, dass viele zur Konferenz kommen und wir werden nicht beleidigt sein, wenn sie sich hier auch als Touristen umschauen. Im Gegenteil, ich möchte die deutschen Mathematiker ermutigen: Kommt nicht nur für die Konferenztage, besonders diejenigen, die noch nie in Jerusalem waren, sollten vorher kommen und danach noch bleiben. Es ist nicht nur die Geschichte, die Jerusalem so interessant macht, auch die Gegenwart. Hier versammeln sich so viele verschiedene Kulturen und Religionen, Musli- me, Christen, Juden. Es gibt manchmal Spannungen, aber es gibt auch viel Kooperation - schließlich leben die Menschen zusammen. Wenn man durch die alten christlichen, muslimischen und jüdischen Bezirke der Stadt läuft, ist das wirklich faszinierend. Man kommt von einem ins andere Viertel und hat den Eindruck, man sei plötzlich in einem anderen Land.

Wird es ein touristisches Begleitprogramm geben?

Ja, wir planen verschiedene Angebote, die meisten davon außerhalb der Stadt. Denn in Jerusalem kann man wunderbar selbst herumlaufen oder an einer Führung teilnehmen. Es wird Ausflüge nach Masada geben, eine antike Festung an den Ufern des Toten Meeres, und nach Caesarea und Akkon, beides Hafenstädte am Mittelmeer. Diese Orte gehören alle zum Unesco-Weltkulturerbe. Manche werden vielleicht auch Gefallen daran finden, die intellektuellen Aktivitäten auf der Konferenz mit der Teilnahme am Jerusalem Marathon zu verbinden, der direkt im Anschluss an die Konferenz am Freitag, den 12. März, stattfindet.

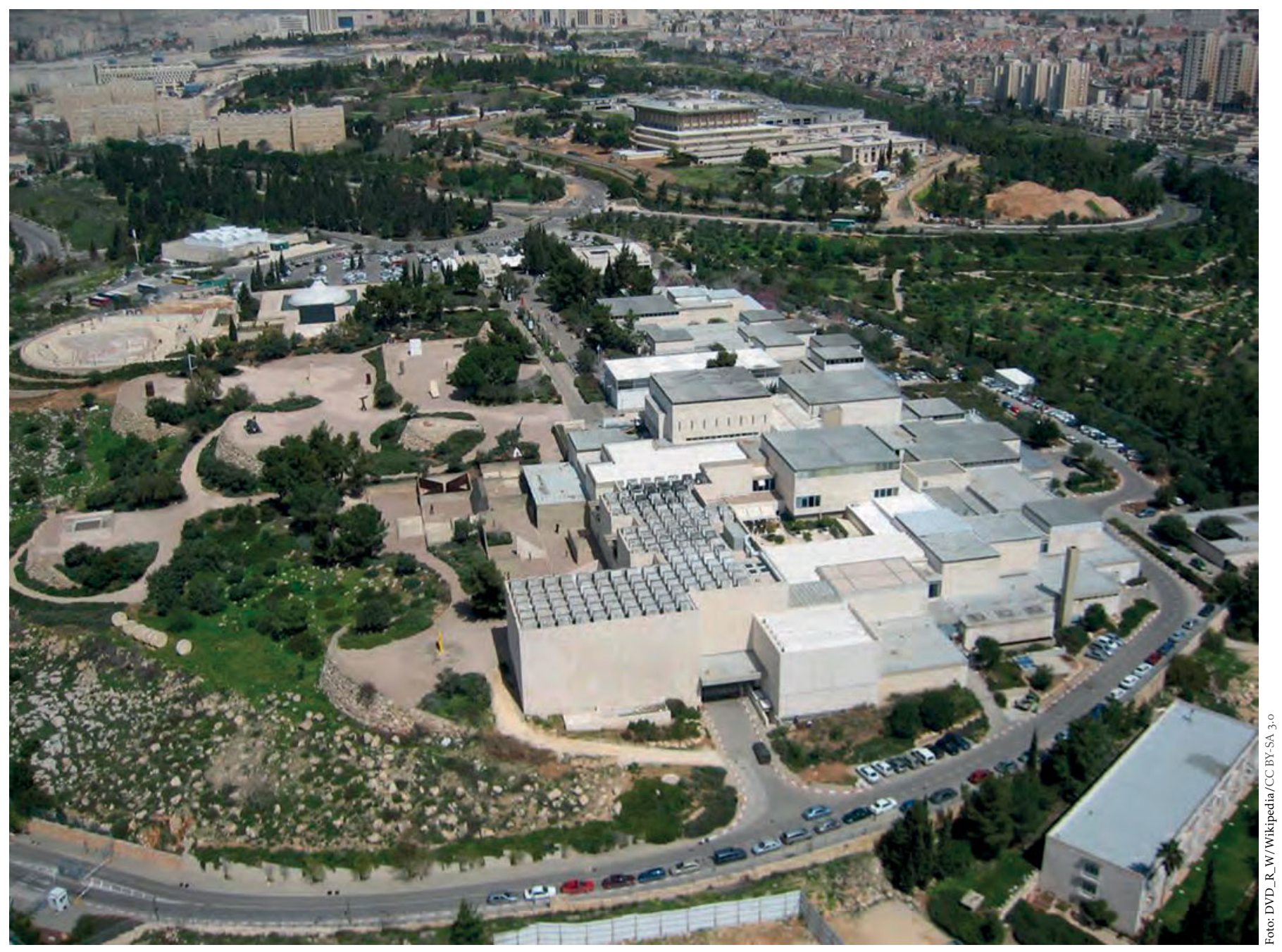

Luftbild von Givat Ram, im Vordergrund das Israel Museum, im Hintergrund die Knesset 


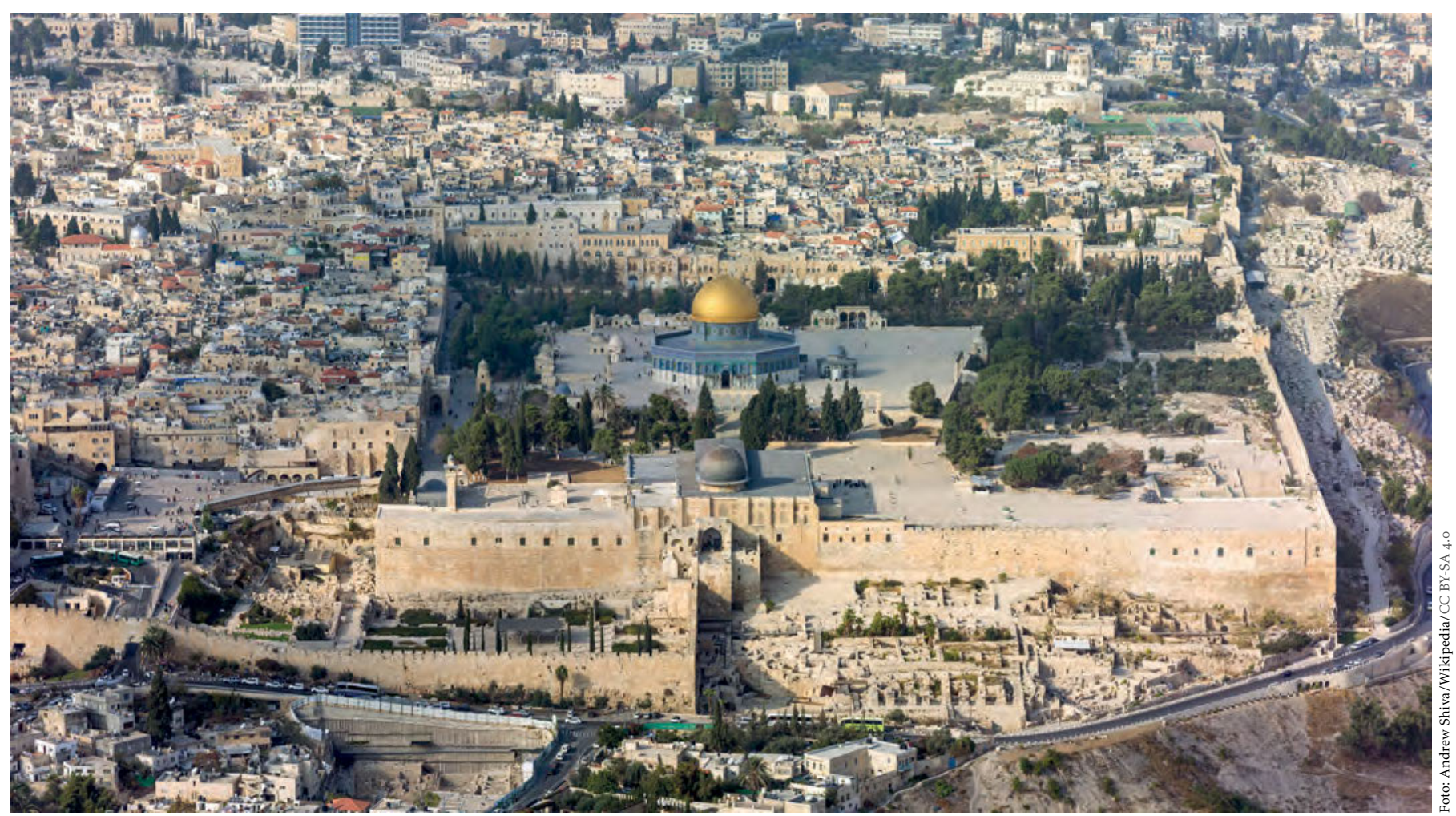

Luftbild des Tempelbergs in Jerusalem

Was erwarten Sie sich von dieser ersten israelischdeutschen Mathematiker-Konferenz?

Kurz gesagt - ich hoffe natürlich auf eine spannende Konferenz mit vielen guten Gesprächen in einer entspannten Atmosphäre. Aber tatsächlich habe ich langfristige Erwartungen: Ich hoffe, dass man sich später an eine Konferenz erinnern wird, die die Kooperation zwischen beiden Ländern vorangetrieben hat. Es gibt hier in Jerusalem die GermanIsraeli Foundation for Scientific Research and Development (GIF) und wenn hier in Zukunft mehr Anträge eingereicht werden, dann wird das ein Erfolg dieser Konferenz gewesen sein.

Noch eine Frage zum Schluss: Warum sollte man diese Konferenz auf gar keinen Fall verpassen?

Diejenigen, die bereits Beziehungen zu Israel pflegen, werden sich über einen erneuten Besuch freuen und ihre Be- ziehungen stärken. Aber eine wirklich gute Gelegenheit wird die Konferenz für all jene sein, die noch gar keine Verbindung nach Israel haben. Entschuldigen Sie, dass ich wieder so unbescheiden klinge, aber wir haben hier so viele Mathematiker und so viele leistungsstarke Mathematiker, dass die Konferenz den Teilnehmern viele Möglichkeiten der Zusammenarbeit eröffnen wird. Mathematiker lieben es zusammenzuarbeiten. Und die Konferenz wird solchen zukünftigen Kooperationen einen Schub verleihen.

Herr Lubotzky, ich danke Ihnen für dieses Gespräch.
Konferenz-Webseite:

http://u.math.biu.ac.il/ vishne/Conferences/IMU-DMV-2021/ 